\title{
Giant Cell Arteritis in Association with Posterior Circulation Strokes: Report of Two Cases with Normal Erythrocyte Sedimentation Rate
}

\author{
Joel C Morgenlander MD ${ }^{1 *}$ and Sarah Neuhaus MD $^{2}$ \\ ${ }^{1}$ Duke Departments of Neurology and Orthopaedic Surgery, Neurology APP Residency Program, USA \\ ${ }^{2}$ Duke Pediatric Neurology, USA
}

*Corresponding author: Joel C Morgenlander MD, Professor, Duke Departments of Neurology and Orthopaedic Surgery, Director, Neurology APP Residency Program, Director, Duke Sports Neurology Clinic, USA.

To Cite This Article: Joel C Morgenlander MD, Giant Cell Arteritis in Association with Posterior Circulation Strokes: Report of Two Cases with Normal Erythrocyte Sedimentation Rate. 2020 - 7(3). AJBSR.MS.ID.001145. DOI: 10.34297/AJBSR.2020.07.001145.

Received: 眥 February 05, 2020; Published: 眥 February 14, 2020

\begin{abstract}
Stroke can be a serious, potentially fatal complication of giant cell arteritis (GCA). GCA and its relationship with ischemic stroke has been extensively studied and clinically investigated; however, a reliable biomarker or clinical manifestation has not yet been identified to detect those patients who are at increased risk of developing brain infarction. In this case series, GCA as the primary etiology of posterior circulation stroke is discussed in two patients with headache and stroke as initial presenting symptoms. Because of unremitting headache without a specific etiology recognized, GCA was considered. Both patients had temporal artery biopsy (TAB) proven GCA, despite normal erythrocyte sedimentation rate (ESR). We make the case to support obtaining TAB in populations at risk for GCA, regardless of inflammatory markers, if headache is a prominent presenting symptom at the time of stroke.
\end{abstract}

Keywords: Temporal arteritis; Giant cell arteritis; Posterior circulation; Ischemic stroke

\section{Introduction}

We describe two patients who presented with symptoms consistent with posterior circulation stroke and the prominent clinical feature of headache. Neuroimaging confirmed ischemic infarction of the posterior circulation, as seen on diffusion-weighted sequences on magnetic resonance imaging (MRI). Both patients had normal ESR on presentation and were ultimately diagnosed with Giant Cell Arteritis (GCA) via temporal artery biopsy (TAB); both responded well to oral steroids with resolution of symptoms once treatment was initiated.

\section{Case 1}

The patient is an 83-year-old male with hypertension, polycythemia, benign prostatic hypertrophy and macular degeneration who presented with a four-day history of persistent headache along with episodic vertigo, gait ataxia, falls, left sided facial/tongue numbness and dysarthria. Patient endorsed persistent pain over his left eye with associated frontal headache. He denied jaw claudication, scalp tenderness or myalgias.
Neurological exam revealed altered pinprick sensation in the left face and left hand, along with tremor in the right arm and an ataxic gait. Vision was compromised secondary to macular degeneration. Strength was intact. Laboratory studies revealed elevated hemoglobin of 19, hematocrit of 56 along with LDL of 149 and hemoglobin A1c of 6.0.

Non-contrast head CT was normal. MRI brain showed two areas of abnormal diffusion restriction in the left posterior parietal lobe and the right cerebellar hemisphere; areas were consistent with acute infarct (Figure 1-A). Magnetic resonance angiography (MRA) of the head and neck revealed significant vascular disease including 75-80\% left proximal internal carotid artery (ICA) stenosis, left supraclinoid ICA tandem lesions and 65\% short segment stenosis of the left vertebral artery. Vertebrobasilar strokes were thought to be possibly related to vertebral artery disease. Transthoracic echocardiogram was normal, revealing only mild left ventricular hypertrophy. Telemetry showed no evidence of arrhythmia. The etiology of stroke was initially attributed to diagnosis of 
polycythemia, however additional genetic studies were negative, including JAK2 V617F mutation or to vertebrobasilar disease.

The patient had persistent headache and constant pain over his left eye/temporal area. Temporal pulses were asymmetric, and the concern was ultimately raised for temporal arteritis in the setting of known posterior circulation stroke. ESR was obtained and found to be normal. Based on persistent headache and suspicion for GCA, patient was started on prednisone $60 \mathrm{mg}$ daily, which immediately resolved the headache symptoms. A temporal artery biopsy was obtained soon after, with pathology consistent with intimal and medial scarring with chronic inflammation, findings histologically compatible with temporal arteritis.

\section{Case 2}

The patient is a 66-year-old male with hypertension, hypercholesterolemia and depression who presented after sudden onset of severe headache, vertigo, diplopia, diaphoresis, photophobia and blurred vision. Headache was described as a sharp pain radiating from the back of the head; severe vertigo led to persistent nausea with emesis. Symptoms were exacerbated by change in position and improved in supine positioning with eyes closed. He denied jaw claudication, scalp tenderness or myalgias. Neurologic exam was remarkable for horizontal nystagmus in both right and left gaze, up-beat nystagmus in up-gaze and mild gait ataxia. Motor and sensory exam was normal. Labs were unremarkable outside of leukocytosis to 18 . Non-contrast CT scan of the head was unremarkable. MRI brain revealed diffusion-weighted changes in the mid-pons (Figure 1-B). Pontine stroke was initially thought to be secondary to small vessel disease. Pharmacological management of vertigo with diphenhydramine, lorazepam, ondansetron and meclizine were attempted, yet unsuccessful. Transthoracic echocardiogram demonstrated mild left ventricular hypertrophy, but no other significant abnormalities and cardiac telemetry monitoring was negative for arrhythmia.

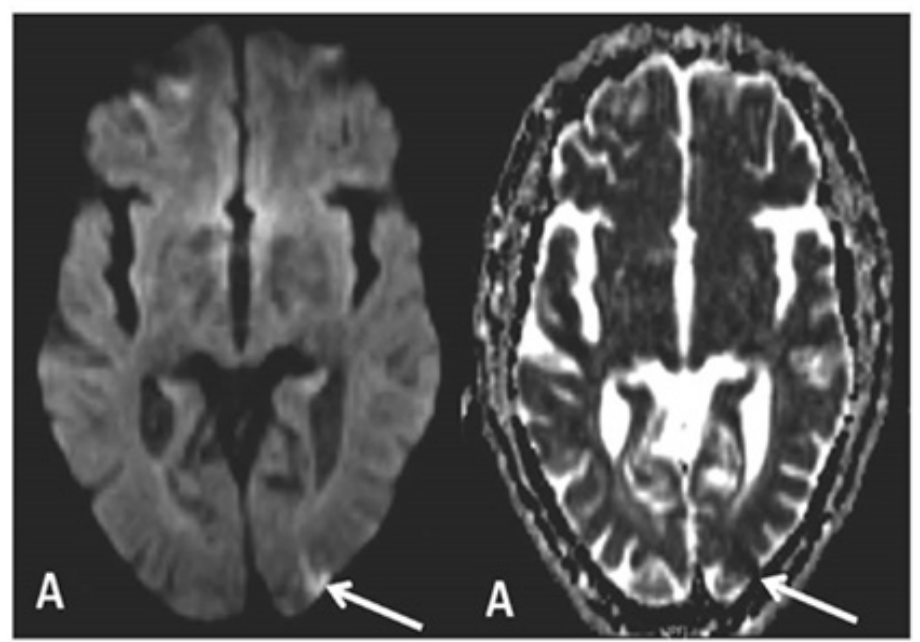

Figure 1-A: MRI imaging of patient 1, showing evidence of acute infarct in the left posterior parietal lobe on DWI and ADC sequences.

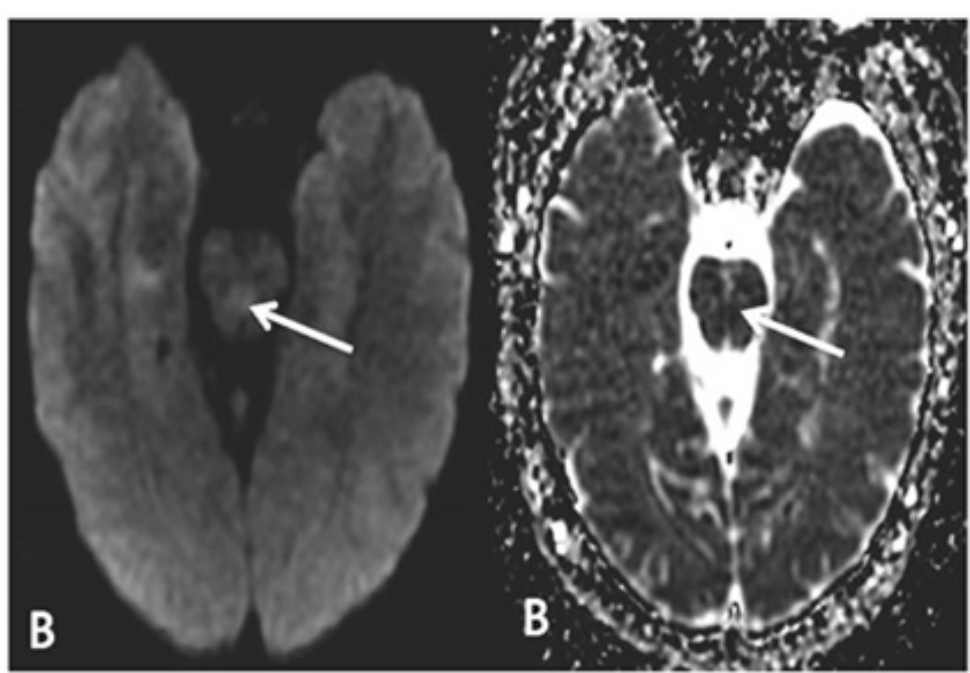

Figure 1-B: MRI imaging of patient 2, showing DWI and corresponding ADC abnormality in the mid pons, representing acute infarct. 
Patient had intermittent dizziness and severe headaches throughout admission, which continued after discharge. He denied jaw claudication or scalp tenderness. There were no new neurologic symptoms, however persistent headaches without explanation were concerning. ESR was obtained to rule out temporal arteritis and found to be normal on two separate occasions. CT angiogram of the head and neck was obtained to rule out vertebral dissection as an etiology of headache but was negative for evidence of dissection. Due to continued headaches, temporal artery biopsy was obtained to definitively rule out temporal arteritis. Biopsy ultimately showed evidence of GCA despite normal ESR. Patient was then started on oral prednisone $60 \mathrm{mg}$ daily, which prompted immediate resolution of headache symptoms.

\section{Discussion}

Previous studies have shown that approximately 4-6\% of patients with GCA experience transient ischemic attack (TIA) or stroke at some point during their illness [1-4]. Retrospective review has also determined that as many as $22.5 \%$ of patients with GCA do not have elevated ESR at time of presentation or diagnosis [5] Medicine still lacks a reliable biomarker or clinical manifestation to enable reliable identification, selection and early detection of those patients who are at elevated risk to develop brain infarction in the setting of GCA [6].

TAB would be specifically warranted in older patients with known posterior circulation stroke and severe headache of unknown cause. According to recent literature reviews, patients manifesting vision loss have an increased risk of stroke $[7,8]$. The mechanism of GCA and posterior circulation stroke is reminiscent of the relationship between anterior circulation stroke and arteritis of cerebral vessels. GCA most commonly affects the tissues of the vertebral arteries more than the internal carotid arteries, especially the extradural portion, due to increased density of elastic tissue [9].

\section{Conclusion}

Careful consideration of the diagnosis of GCA should be given in the appropriate clinical context. More specifically, older patients with evidence of new posterior circulation stroke and a key clinical feature of persistent headache of unknown cause should warrant thorough investigation, including $\mathrm{TAB}$, to definitely rule out GCA. Evidence to support obtaining inflammatory markers, such as ESR and C-reactive protein, as part of the routine workup for patients presenting with TIA or stroke-like symptoms and headache is well known and accepted. However, with these cases, we aim to emphasize the importance of obtaining a pathological confirmation via TAB in populations at risk for GCA, regardless of inflammatory markers, especially if headache is prominent. Without biopsy as supportive evidence, it can be difficult to commit to temporal arteritis as the primary etiology of stroke, thus missing an important and treatable disease process.

\section{References}

1. Caselli RJ, Hunder GG, Whisnant JP (1988) Neurologic disease in biopsyproven giant cell (temporal) arteritis. Neurology 38(3): 352-359.

2. Kumar A, Costa DD (2007) Insidious posterior circulation stroke with rapid deterioration due to vertebral giant cell arteritis. Age Ageing 36(6): 695-697.

3. Mclean CA, Gonzales MF, Dowling JP (1993) Systemic giant cell arteritis and cerebellar infarction. Stroke 24(6): 899-902.

4. González Gay MA, Blanco R, Rodríguez Valverde V, Martínez Taboada VM, Delgado Rodriguez M, et al. (1998) Permanent visual loss and cerebrovascular accidents in giant cell arteritis: predictors and response to treatment. Arthritis Rheum 41(8): 1497-1504.

5. Ellis ME, S Ralston (1983) The ESR in the diagnosis and management of the polymyalgia rheumatica/giant cell arteritis syndrome. Ann Rheum Dis 42(2): 168-170.

6. Kawasaki A, V Purvin (2009) Giant cell arteritis: an updated review. Acta Ophthalmol 87(1): 13-32.

7. Berger CT, Wolbers M, Meyer P, Daikeler T, Hess C (2009) High incidence of severe ischaemic complications in patients with giant cell arteritis irrespective of platelet count and size, and platelet inhibition. Rheumatology Oxford 48(3): 258-261.

8. Nordborg E, C Nordborg (2003) Giant cell arteritis: epidemiological clues to its pathogenesis and an update on its treatment. Rheumatology 42(3): 413-421.

9. Salvarani C, Giannini C, Miller DV, Hunder G (2006) Giant cell arteritis: Involvement of intracranial arteries. Arthritis Rheum 55(6): 985-989. 